rev.relac.int.estrateg.segur.10(1):159-179,2015

\title{
IZQUIERDA Y PODER ELECTORAL EN BOGOTÁ: UNA RECONSTRUCCIÓN HISTÓRICO-COYUNTURAL (2004-2012)•
}

\author{
Julián Darío Bonilla Montenegro**
}

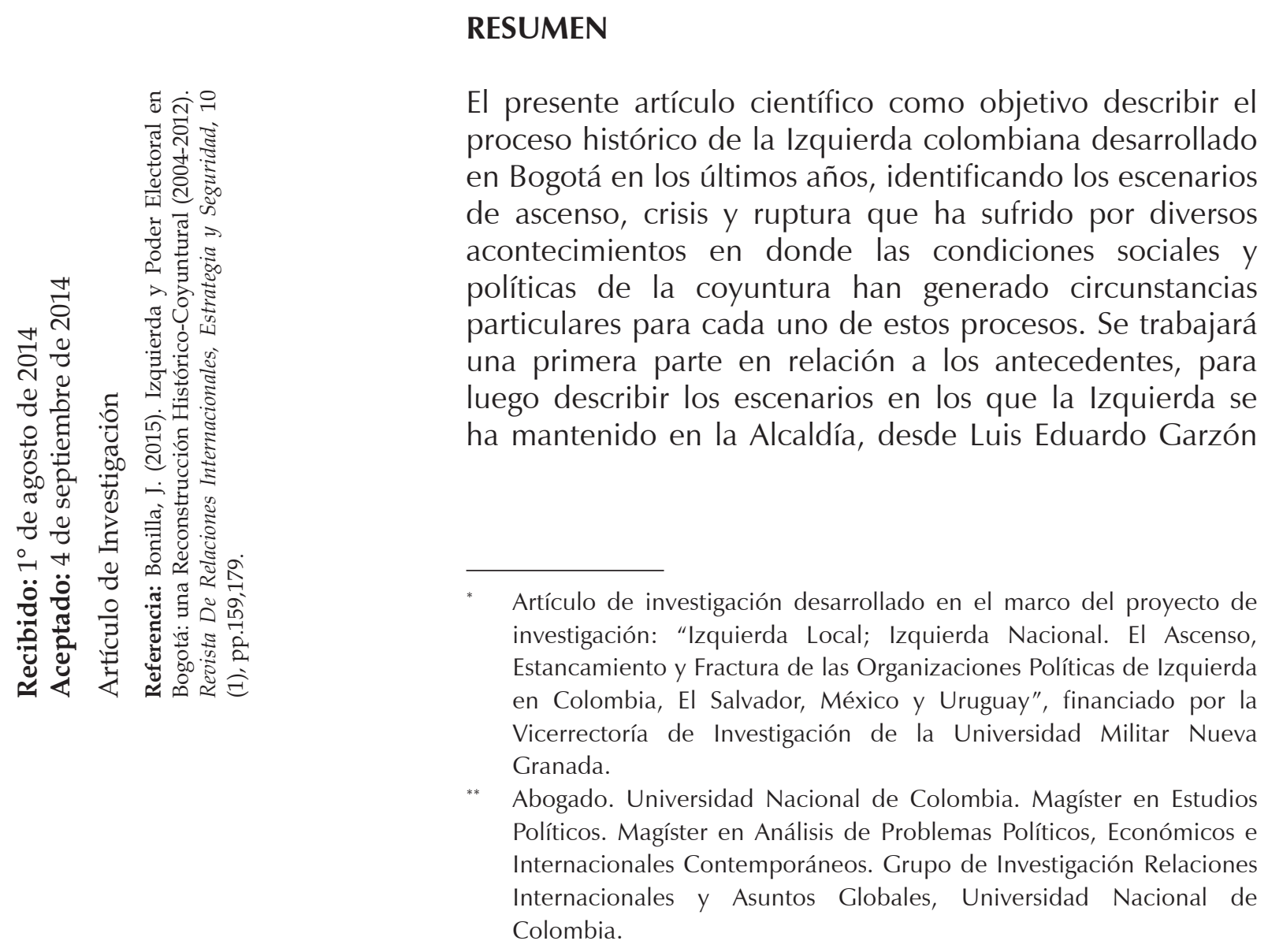


(2004-2007) hasta Gustavo Petro Urrego (desde 2012), pasando por la fase más crítica de la Alcaldía de Samuel Moreno Rojas - Clara López Obregón (2008-2011). La base del proceso es la construcción desde el análisis de coyuntura y la recolección de información se basó esencialmente en el manejo de minería de datos (web mining), recurriendo a diversas fuentes de prensa que han analizado dicho proceso.

Palabras clave: Bogotá, Elecciones, Historia, Izquierda.

\title{
LEFT MOVEMENTS AND ELECTORAL POWER IN BOGOTA: A SITUATIONAL-HISTORICAL CONSTRUCTION (2004 -2012)
}

\begin{abstract}
This article has as a main objective, to describe the historical process of the Colombian left movement developed in Bogota during the last years, identifying the stages of ascent, crisis and break up that the left has suffered by a variety of events where the situational's social and political conditions have generated particular circumstances in each process. It will be worked a first part related with the precedents, and then it will describe the stages in which the left movements have been maintained in the mayor's office, since Luis Eduardo Garzón (2004-2007) up to Gustavo Petro Urrego (since 2012), crossing the criticized phase during the Samuel Moreno - Clara López Obregón's government (2008-2011). The base of this process is the construction based on the situational analysis and the access of the information was built essentially with the management of web mining, using press sources that have analyzed this process.
\end{abstract}

Keywords: Bogotá, Elections, History, Left Movements.

\section{MOVEMENTOS DE ESQUERDA E PODER ELEITORAL EM BOGOTA: UMA RECONSTRUÇÃO HISTÓRICA - COJUNTURAL (2004-2012)}

\section{RESUMO}

O presente artigo tem como objetivo descrever o processo histórico da esquerda colombiana nos últimos anos, identificando o cenários de ascenso, crises y ruptura que tem sofrido por diversos sucessos onde as condições sociais e políticas da cojuntura têm gerado circunstâncias particulares para cada uno destes processos. O trabalho começará em uma primeira parte em relação aos antecedentes, para depois descrever os cenários nos que a esquerda tem mantido na Prefeitura, desde Luis Eduardo Garzón (2004-2007) até Gustavo Petro Urrego (desde 2012), passando a criticada fase durante o governo de Samuel Moreno - Clara López Obregón (2008- 
2011). Na base do processo é a construção desde a análise de cojuntura e a recoleção da informação foi feita de maneira essencial no manejo da mineração de dados (web mining), recorrendo a fontes imprensas que tem analisado este processo.

Palabras claves: Bogotá, Eleções, História, Movementos de Esquerda.

\section{INTRODUCCIÓN}

Uno de los fenómenos políticos que más ha llamado la atención en los últimos años al analizar la región de América Latina, es lo que se ha denominado el giro a la Izquierda, relacionado con dirigentes políticos de ésta corriente ideológica que han alcanzado los más altos cargos de poder político (por los sistemas políticos presentes se habla de la Presidencia) en diversos países de la región.

En estos casos, se hace mención a lo acontecido en los gobiernos de Hugo Chávez en Venezuela, Evo Morales en Bolivia, Rafael Correa en Ecuador o Daniel Ortega en Nicaragua, cuando se hace referencia a una izquierda de corte radical en contraposición a lo que se ha presentado en los casos de Luiz Inácio Lula da Silva -Dilma Rouseff en Brasil o la continuidad del Frente Amplio uruguayo con Tabaré Vásquez Rosas- José Mújica en Uruguay, quienes son algunos representantes de la corriente de la denominada Izquierda Socialdemócrata, que todavía acepta las políticas del mercado para el sostenimiento de la economía y, al mismo tiempo, no han recurrido a estrategias constitucionales para lograr mantenerse en el poder.

Escenario que ha permitido recordar lo expresado por Eagleton (citado por Hamburger, 2014), "todavía existen muy buenas ideas de izquierda en todas partes, y un no menos fértil y sugerente corpus de trabajo sobre cómo podría ser una economía socialista" (p.144).

Sin embargo, ¿qué ha acontecido en Colombia en los últimos años? A diferencia de otros países de la región, y en alguna medida en sintonía con lo que se ha presentado en Ciudad de México, la Izquierda en Colombia ha logrado posicionarse en el segundo cargo más importante de elección popular del país: la Alcaldía Mayor de Bogotá, pero no ha podido lograr dar el salto hacia la Presidencia de la República.

A diferencia de México, donde el Partido de la Revolución Democrática se ha sostenido sobre una base política y electoral estable, en el caso colombiano es posible observar una fractura que contradice la intención de unidad de la izquierda a la que tanto aludían los miembros de los partidos y movimientos políticos de esta tendencia ideológica, generando una situación de estancamiento e inclusive de retroceso para la Izquierda colombiana, sin que en este momento pueda considerarse un panorama promisorio para los próximos años. 
Condiciones que demuestran, en parte, que la organización estatal colombiana no ha permitido generar verdaderos procesos para transformar las problemáticas que históricamente existen en Colombia (Mendoza, 2006) y que son la base de un discurso tradicional de la Izquierda política.

Con base en lo anterior, este artículo busca establecer desde un análisis de tipo coyuntural, el resolver la problemática acerca de cuáles son los acontecimientos más relevantes en los que ha participado la Izquierda colombiana a nivel electoral en las elecciones a la Alcaldía Mayor. Lo anterior permite una primera hipótesis validable mediante el trabajo de recolección de fuentes a través de la minería de datos, la cual consiste en establecer que la articulación de los acontecimientos dentro del escenario electoral en Bogotá, impulsa las tendencias de las masas electorales para configurar una relación de carácter coyuntural que incide en los triunfos de la Izquierda política desde el año 2003 hasta la fecha.

A pesar de que se considera que existe la Izquierda en el poder capitalino desde el año 2004, ésta no ha sido estable y no ha sido posible un verdadero proceso de ascenso con miras a las elecciones de tipo nacional. Para poder demostrar lo anterior, se construirá primero un referente con base en los antecedentes a este proceso en donde también participaron candidatos de Izquierda, para analizar con más detalle los casos de Luis Eduardo Garzón (2004-2007), Samuel Moreno - Clara López (2008-2011) y Gustavo Petro Urrego (2012 - actualmente).

\section{PRECISIONES CONCEPTUALES Y METODOLÓGICAS: ANÁLISIS HISTÓRICO COYUNTURAL}

Vale la pena entonces aclarar las razones para construir éste análisis histórico-coyuntural pues, de acuerdo con el profesor de la Universidad Nacional de Brasilia, Antonio Ramalho, "por razones didácticas, los historiadores tienden a organizar en periodos la interpretación de lo que consideran los acontecimientos más importantes del pasado, En rigor, las cosas se presentan a los analistas en una especie de continuum, respecto del cual, poco a poco, se construyen interpretaciones, con la ayuda de diferentes métodos de investigación" (Ramalho, 2007, p.435).

Lo anterior también demuestra que el análisis histórico-coyuntural es un asunto de permanente retroalimentación (Fazio, 2007), que se lleva a cabo a partir de la reconstrucción de huellas, indicios y vestigios que se asocian con el escenario que se pretende estudiar. Siguiendo al profesor Hugo Fazio "son los soportes sobre los cuales se sostiene y en los que se valida la correspondiente argumentación, narración y/o (sic) descripción" (2007, p.191), mediante la constante interrelación que existe entre los elementos constructores de un proceso histórico, que en este caso se construye sobre una temporalidad de mediana duración, sobre la base de una coyuntura que establece "una situación que resulta de un encuentro de circunstancias y que se considera como el punto de inicio de una evolución o una acción" (Fazio, 1998, p.54). 
Asimismo, al ser un trabajo de tipo descriptivo, se enfoca en la revisión documental, que ofrece información detallada sobre los acontecimientos que generan determinadas tendencias durante el periodo de estudio propuesto en la investigación (Bernal, 2006). Es así que, de acuerdo con Gerard Radnitzky (2000), la investigación debe ayudar al investigador a relacionarse con otras investigaciones tanto en un medio intelectual como respecto de los intereses externos, incluyendo las fuerzas sociales y políticas.

Dicha función es la naturaleza misma de la investigación, que se compone de elementos humanos, actividades o procesos y productos siendo pues el resultado de una práctica en el constructo del proceso investigativo. Por esta razón, y para lograr mejores espacios en este análisis histórico reciente, se hace necesario tener en cuenta una búsqueda constante de información por parte de los medios de comunicación virtuales.

Es por lo expuesto en el párrafo anterior que se reconoce que en los últimos años los artículos de prensa y documentos académicos, confirman la necesidad de aplicar técnicas de Web Content Mining o Minería de Datos, con el fin de extraer de la información útil proveniente de la Web, para los intereses de la investigación, reconociendo los patrones generales de búsqueda sobre las temáticas desarrolladas en la investigación, logrando un análisis correcto de acuerdo a los criterios que se exigen en un trabajo de este estilo.

En algunas partes del proceso de investigación se establecen criterios de búsqueda para la recuperación de información, en tanto que otros, especialmente los artículos indexados, se manejan los criterios de búsqueda y procesamiento de información mediante la vista de base de datos (De Gyves, s.f.).

Para el ejercicio investigativo en este artículo se efectúo una labor de búsqueda de información especialmente en páginas web de carácter nacional, reconocidas nacional e internacionalmente ${ }^{1}$, que han cubierto de manera permanente diversos procesos del acontecimiento político nacional y por esta razón, ofrecen información concreta sobre el periodo esencial de estudio descrito.

Las razones para su enfoque se deben a las revisiones previas que se han realizado y de éstas se ha conseguido la mayor información hasta el momento. Además, se considera que debido al cada vez mayor flujo de información al que se puede acceder mediante los medios virtuales, es conveniente establecer un criterio selectivo concreto para la búsqueda de los datos requeridos en esta construcción histórica, teniendo en cuenta el fenómeno de la dispersión de la información.

1. Las páginas trabajadas fueron: Revista Semana (www.semana.com), el portal de La Silla Vacía (www.lasillavacia.com); así como El Espectador (www.elespectador.com) y El Tiempo (www.eltiempo.com). Se podrá también encontrar información de otras páginas Web pero estas son en un número reducido en comparación con la información recopilada de los dominios referidos. 
Pues "del número ingente de páginas que aparecen al buscar algo, sólo muy pocas llegan a ser verdaderamente útiles" (Alted y Sánchez, 2006, p.257). Para reducir el número de páginas y especificar los criterios de búsqueda de la información, concentrándose en las temáticas que se extraen del trabajo, se desarrolló la actividad mediante el uso del metabuscador Copérnico, el cual permite buscar grupos de noticias de acuerdo a los criterios establecidos particularmente.

Antes de dar inicio a los antecedentes, vale la pena hacer una precisión de carácter conceptual: por diversas razones se ha considerado que las alcaldías de la Capital, desde 2003 a la fecha, son gobiernos de Izquierda, aun cuando directamente no se pueda endilgar si realmente hacen parte de esta categoría de accionar ideológico.

Por ejemplo, el origen de Samuel Moreno proviene más de una partido de tendencia populista que directamente vinculado a la Izquierda en su momento (como es el caso de la Alianza Nacional Popular, ANAPO). Asimismo, desde el Polo Democrático Alternativo (PDA) se ha tratado de construir la idea de manera sistemática que Gustavo Petro, del Movimiento Progresista (MP), no ha ejecutado una obra de gobierno directamente vinculada a las políticas de Izquierda, sino que ha llevado a cabo la implementación constante de prácticas de corte neoliberal.

La razón básica para considerarlos de Izquierda exclusivamente para éste artículo, se debe a las razones que presentaron durante sus campañas políticas y por los estatutos que constituyen sus partidos o movimientos políticos, o por la manera como las fuentes sobre las que se trabajó la información las catalogaron. Sobre este último punto, también es preciso aclarar que, aunque sus estatutos lo mencionen, no se considerará que el Partido Liberal (PLC) sea de una tendencia política de Izquierda, debido a muchas de sus líneas de acción, que van en contravía de los postulados básicos de esta corriente ideológica. Por esta razón, no se considerarán como gobiernos de Izquierda local los casos de Juan Martín Caicedo Ferrer o Jaime Castro Caicedo.

\section{ANTECEDENTES DEL PROCESO HISTÓRICO DE LA IZQUIERDA EN BOGOTÁ}

Bogotá es el referente nacional de la manera como han surgido fuerzas políticas alternativas distintas a los grupos políticos tradicionales, o los que actualmente (debido a las complejidades que han generado los últimos acontecimientos) han controlado el poder desde las élites políticas.

La situación ocurre desde mediados de los 90s, después de un proceso que incluyó en primer lugar el Acto Legislativo 01 de 1986 que permitió por primera vez la elección de alcaldes mediante participación democrática popular a través de los consensos bipartidistas que todavía prevalecían en esa época (Cepeda, s.f.), al sistema establecido en la Constitución Política de 1991, la cual dio cabida a la obligatoriedad de la elección en todos los cargos, fueran estos uninominales (alcalde, gobernador) o plurinominales (diputados, concejos) en los departamentos y municipios del país. 
Es a partir de este momento en que en Colombia se empezó a mencionar la existencia de un sistema marcadamente personalista (Ministerio del Interior, 1999), alejado de los sistemas políticos partidistas tradicionales y en donde las condiciones de movilización de las masas dependen de consideraciones ajenas a una supuesta ideología o pertenencia política. La incertidumbre acerca de las consecuencias que se generarían dentro del sistema electoral no fue contemplada.

Al comienzo de los cambios en el sistema electoral los primeros alcaldes de la capital fueron miembros de los partidos tradicionales, Partido Conservador Colombiano (PCC), con Andrés Pastrana Arango y el PLC, bajo las gestiones de Juan Martín Caicedo y Jaime Castro (Dávila, 1992). No fue sino hasta la llegada del matemático Antanas Mockus en 1995 que se contó con la presencia de un outsider ${ }^{2}$, que se enfrentó contra candidatos del bipartidismo y logró el triunfo gracias a la construcción en torno a él, de una amalgama de propuestas así como la imagen alternativa que necesitaba Bogotá en ese momento ${ }^{3}$.

Durante este primer periodo a analizar, la participación de movimientos vinculados con la Izquierda puede considerarse minoritaria, pues nunca alcanzaron un reconocimiento mediático ni electoral durante el ejercicio de estos procesos, pues generalmente solamente se reconoce la participación que tuvo María Eugenia Rojas a nombre de ANAPO en 1988, así como también Clara López Obregón, quien para el año 1988 efectuó su primera participación electoral, como candidata a la Alcaldía de Bogotá por Unión Patriótica (UP) (Registraduría Distrital, 1988). Después, en las elecciones del año 1990, fueron candidatos desde la Izquierda del espectro político, el recién desmovilizado comandante del M-19, Carlos Pizarro y el veterano político Carlos Romero a nombre de la UP (Registraduría Distrital, 1990).

Es en el año de 1992, donde se puede observar una mayor cantidad de candidatos que representan la Izquierda política, pues, de acuerdo con la información suministrada por las entidades oficiales, fueron 4 los candidatos que representaron movimientos o partidos políticos de esta tendencia ideológica.

Así, para las elecciones que ganó Jaime Castro fueron candidatos Carlos Alonso Lucio, a nombre de una disidencia del M-19; Jaime Caicedo en representación de la UP; Gerardo Ardila, quien provenía de una fracción de la Alianza Democrática (AD) M-19 y finalmente, Rosa Celia Lemus, en representación del Partido Socialista de los Trabajadores (PST) (Alcaldía Mayor de Bogotá, 2001).

2. Se puede entender al outsider como aquel candidato que ya necesita de la clase política tradicional, generalmente vista como contaminada. Este nuevo político entra en al juego electoral "bajo la forma de una imagen, no bajo la forma de cuerpos en lugares de oscuras rocas políticas" (Landi, 1995, p, 204)

3. Una descripción sobre cómo era la percepción de Bogotá para mediados de 1990 y la construcción simbólica que se llevó a cabo en torno a Antanas Mockus primero y Enrique Peñalosa después, se encuentra en el documental danés, Bogota Change (Dalsgaard, 2009). 
A partir de ese entonces, las dinámicas de los actores para alcanzar este cargo mediante la competencia electoral, han logrado atraer la atención de otros grupos políticos y se ha consolidado la posibilidad de permitir la participación de nuevas fuerzas políticas, sin que las candidaturas de los partidos oficiales tengan una aceptación clara dentro del electorado ${ }^{4}$.

Luego de Mockus fue el turno para Enrique Peñalosa, quien a pesar de tener un origen familiar de índole liberal y en sus antecedentes políticos, se retiró de dicho partido para participar bajo la imagen de ofrecer una alternativa independiente en las elecciones de 1997. Su movimiento se llamó Por la Bogotá que Queremos y tuvo como principal contendor a Carlos Moreno de Caro, quien, al igual que Peñalosa, no se encontraba dentro de un partido político pues su participación se construyó en torno al movimiento Defensa Ciudadana, aun cuando su origen era el PCC. En esa ocasión también se presentó como candidato de Izquierda Gustavo Petro Urrego, todavía a nombre de la Alianza Democrática M-19 (El Tiempo, 7 de agosto, 1997), movimiento político fruto de la desmovilización efectuada iniciada la década de 1990 por parte de la Guerrilla del Movimiento 19 de abril (M-19), del cual Gustavo Petro fue integrante.

El año 2000 fue nuevamente el turno para Mockus. Él había renunciado de manera anticipada a su primer periodo por sus intenciones de aspirar a la Presidencia. Sin embargo, él mismo llevó a cabo una disculpa pública enfrentándose a la candidata María Emma Mejía, quien anteriormente fue la formula vicepresidencial de Horacio Serpa Uribe en las elecciones de 1998 bajo el rotulo del PLC. El triunfo de Mockus consolidó la tendencia acerca de la independencia política así como las expectativas "en torno a la continuidad de las políticas del gobierno distrital, sustentadas en la recuperación del espacio público y el crecimiento urbano desde la planificación" (Otalora, s.f., p.31), iniciada en la administración de Peñalosa.

En ese momento, la crisis de los partidos políticos tradicionales fue evidente. Solo hubo una candidatura oficial: William Vinasco Chamorro fue candidato por el PCC y el PLC decidió no participar y dar su apoyo tácito a María Emma Mejía.

Asimismo, de acuerdo a lo que expone Francisco Gutiérrez, se empezó a conformar un autorreconocimiento en la arena electoral, ajena a la tradición política habitual (Gutiérrez, 1995).

Son estos una serie de acontecimientos que pueden interpretarse por la disposición que ha asumido el elector bogotano con el fin de:

4. Candidatos con una larga tradición dentro del bipartidismo han preferido lanzar candidaturas de manera independiente. Además del caso de Peñalosa, se encuentra, por ejemplo, la candidatura de María Emma Mejía en 2000 a nombre del movimiento María Emma Firme Bogotá o recientemente Jaime Castro quien en el 2011 fue candidato por el Movimiento de Autoridades Indígenas de Colombia.

5. Peñalosa fue Representante a la Cámara por el PLC y su padre, Enrique Peñalosa Camargo, dirigente de este partido tradicional de Colombia. 
cambiar aquellas estructuras que influyen negativamente sobre las relaciones sociales que configuran las identidades, lo que constituye formas particulares de socialización política en tanto formas de aprendizaje político, manifiesto o no, permanente, deliberado o no, que afecta el comportamiento, las actitudes y las características de la personalidad en lo político (Lozano, 2008, p, 350).

\section{ASCENSO DEL PROCESO POLÍTICO DE LA IZQUIERDA EN LA CAPITAL. ALCALDÍA DE LUIS EDUARDO GARZÓN (2004-2007)}

EEn 2003 llegó el turno para que representantes políticos de la Izquierda en Colombia obtuvieran un fortalecimiento en su imagen política y fuera ésta considerada una opción de poder. La situación se inició al momento de la conformación del Polo Democrático (PD) en 2002, como una alianza política que se aglutinó en torno a la candidatura de Luis Eduardo Garzón, apoyado inicialmente por el partido Frente Social y Político (FSP) de quien él fue parte por su vinculación con el Partido Comunista Colombiano (PC), y la Unión Patriótica, UP) (La Silla Vacía, 23 de noviembre, 2010), y en seguida recibió la adhesión de otros movimientos como el llamado Vía Alterna (VA) que se diseñó en torno a las campañas de Antonio Navarro Wolff al Senado y de Petro a la Cámara de Representantes, así como el partido ANAPO de tendencia populista ${ }^{6}$, junto con la participación de otros actores de oposición ${ }^{7}$.

Garzón, conocido como Lucho, al dejar el FSP se acercó al recién creado Polo Democrático Independiente (PDI) y fue proclamado como su candidato a la Alcaldía de Bogotá, enfrentándose al periodista Juan Lozano, quien en ese momento contaba con el apoyo del ex Alcalde Peñalosa.

Finalmente Garzón obtuvo el cargo que le dio pie a ser comparado con el Presidente de Brasil en aquella época Lula da Silva, debido al origen humilde de ambos y su pasado con el movimiento sindical (Revista Semana, 23 de diciembre, 2002), además de "un giro gradual hacia una visión de conciliación política y social basada en la creencia en un gran pacto entre clases sociales y entre diferentes sectores de la sociedad" (Rodríguez, 2008, p.205), que fue analizado como la posibilidad de que en Colombia se diera el giro a la izquierda, más sin embargo no despertó las expectativas para consolidar un proyecto político debido en parte a la configuración de un partido transversal, con sectores de otras tendencias políticas como el liberalismo y el conservatismo, que terminó por alejarlo de varias de las instancias de decisión dentro de la reciente organización política del PDI (Estrada, 2008).

6. Acerca del proceso histórico de este partido, se considera esencial el trabajo de César Ayala (1995). Es importante saber su configuración pues el siguiente alcalde de Izquierda en Bogotá, Samuel Moreno, es de la línea directa de los fundadores de dicho partido a inicios de la década de los 60.

7. La información se encuentra detallada en Registraduría Nacional del Estado Civil, (6 de julio, 2002) 


\section{CONSOLIDACIÓN DEL PROCESO: ALCALDÍA DE SAMUEL MORENO ROJAS (2008-2011)}

En 2007, la elección a la Alcaldía de Bogotá se circunscribió entre las candidaturas de Samuel Moreno Rojas a nombre del Polo Democrático Alternativo (PDA), partido que surgió luego de la unificación entre el PDI y la coalición de partidos de izquierda denominada Alternativa Democrática (AD), la cual mantuvo una actividad de bancada en el congreso durante el periodo 2002-2006.

De este movimiento hacían parte organizaciones como el Movimiento Obrero Independiente Revolucionario (MOIR), el FSP, Partido Comunitario Opción Siete (PCOS), Unidad Democrática (UD), entre otra serie de movimientos sociales de tendencia comúnmente conocida como Izquierda.

Luego del lanzamiento de la candidatura de Navarro Wolff por parte del $\mathrm{PDI}^{8}$ y de la precandidatura de Carlos Gaviria Díaz a nombre de $\mathrm{AD}^{9}$, se estableció la posibilidad de lograr una consulta abierta entre ambos candidatos a nombre de un mismo partido para establecer cuál de los dos sería candidato presidencial (Basset, 2008).

Posterior a la conformación oficial del PDA para participar en las elecciones nacionales de 2006, se construyó la idea de llevar a cabo una consulta popular para las elecciones a la Alcaldía de Bogotá en 2007 en la que participó Samuel Moreno Rojas (Caracol Radio, 5 de marzo, 2007). El obtuvo la victoria de dicha consulta, marcada por unos muy elevados niveles de abstención y así quedó proclamado como candidato el día 8 de julio de ese mismo año. En ese momento, la posibilidad de unidad se había considerado como un hecho, gracias a los resultados obtenidos en la campaña presidencial de Carlos Gaviria en el 2006, así como la participación de diversos candidatos, todos ellos representando diversas tendencias ideológicas, en la consulta mencionada.

Inclusive, en ese proceso electoral participó la ex Candidata Mejía, en dicha ocasión, bajo la sombra del PDA y contó con la participación de Hernando Gómez Serrano, quien comenzó su actividad proselitista siendo candidato en el año 2000 bajo una coalición de partidos de Izquierda (UP - Vía Alterna, VA), el cual fue el embrión del proceso de consolidación del FSP, siendo esta su primera acción en un proceso electoral relevante dentro del país (Flórez, 2011).

8. Fue designado como candidato mediante una consulta interna llevada a cabo en noviembre de 2004. Se enfrentó a Guillermo Alfonso Jaramillo y a Samuel Moreno Rojas. Éste, aceptó la dirigencia del PDI y anunció a partir de ese momento su intención de ser candidato a la Alcaldía de Bogotá. El Tiempo (13 de noviembre, 2004).

9. Aceptó con insistencia la solicitud que obtuvo desde movimientos como el MOIR, así como la petición personal que le dio el intelectual colombiano Orlando Fals Borda. Durante el segundo semestre de 2004 se inició el lanzamiento de su precandidatura, teniendo como punto final el día 4 de diciembre en Bogotá. 
Al inicio del proceso electoral se descartó que el Alcalde fuera Peñalosa. Sin embargo, durante el transcurso del mismo, comenzó a perder en su intención de voto frente a Samuel Moreno, situación que se consolidó luego del escándalo que se quiso impulsar en contra tanto de la campaña de Moreno Rojas como del PDA tras la respuesta que el entonces candidato dio a una pregunta en un debate llevado a cabo una semana antes de las elecciones hecha por el Ex Alcalde Mockus acerca de la posibilidad de comprar 50 votos para evitar que ganara alguien que pudiera comprar 50.000. La respuesta Sí. No lo dudo ${ }^{10}$, causó tal turbación que incluso llevó a que el entonces Presidente Álvaro Uribe hiciera referencia a ese caso como una forma indirecta de interceder políticamente en contra del en ese momento candidato de la oposición, pues en su parecer, cuando faltaban menos de 7 días para las elecciones, llamó la atención sobre no votar por un candidato apoyado por la guerrilla y que compra votos (El Tiempo, 26 de octubre, 2007).

El acontecimiento descrito generó un efecto contrario, pues formó un descontento político producto del rechazo generalizado que las masas electorales, tanto votantes potenciales como indecisos, construyeron como una forma de expresar una alternativa a los resultados que se plasmaron en contra de las acciones que Samuel Moreno ofreció. Bajo las condiciones del descontento político, se hizo necesario romper con los parámetros que se consideraron opresivos a la decisión libre en el ejercicio electoral y de esta manera se establecieron alternativas como forma de liberación o de protesta.

Por esta razón, el compromiso político de los electores se basó en ese momento más en una forma de protesta en contra de las acciones ${ }^{11}$ que se llevaron a cabo por la respuesta que Moreno ofreció, así también, como en contra de las circunstancias que muchos políticos llevaron en contra de él. Se prefirió reinterpretar la intención de voto y otorgarle un apoyo al PDA como partido de oposición, o inclusive a la tercería que en este momento se conformaba en torno a una segunda candidatura del locutor de radio William Vinasco Chamorro, en esa ocasión, no como candidato del PCC, sino a nombre del Movimiento Afrocolombiano (MA).

Asimismo, es importante mencionar que gracias al triunfo que obtuvo Moreno Rojas sobre Peñalosa, se llegó a pensar en la posibilidad de los avances de los gobiernos de Izquierda a nivel local lograrían frenar el impulso que se presentó en diversos escenarios electorales del país sobre la fuerzas políticas de derecha o conservadoras, generando en el imaginario de los simpatizantes de esta tendencia política, la idea de una verdadera consolidación. Situación que logró ampliar el fuerte entusiasmo de los militantes de los partidos y movimientos de Izquierda colombianos, pero que desconocían la complejidad del desarrollo político que se ha construido en torno a otros procesos electorales y los acontecimientos de crisis y ruptura que llegaron después de este triunfo electoral.

10. La pregunta y la respuesta pueden observarse en YouTube (2007). No se comparte la descripción que se hace del vídeo.

11. Una de esas acciones fue la declaración del presidente Uribe [supra]. 


\section{CRISIS EN LA CONFORMACIÓN DEL PROCESO UNIDAD DE LA IZQUIERDA: CARRUSEL DE LA CONTRATACIÓN Y PRIMERA RUPTURA (2010)}

Después de las elecciones presidenciales en Colombia llevadas a cabo durante el primer semestre de 2010 y en las que participaron Petro como candidato del PDA y Peñalosa, primero como precandidato del recién organizado Partido Verde (PV) y luego como acompañante en varias de las actividades políticas del entonces candidato Mockus, se presentó uno de los acontecimientos que marcaron la pauta en relación con las decisiones que podrían tomar los votantes en el proceso electoral para elegir el próximo alcalde capitalino: el llamado Carrusel de la Contratación. Acontecimiento que empezó cuando fueron presentadas una serie de grabaciones en la emisora Caracol Radio en la que involucraban la presunta negociación con contratistas del Distrito y el Contralor de Bogotá (Caracol Radio, $1^{\circ}$ de febrero, 2011).

El 21 de octubre de ese mismo año, una comisión encabezada por Petro, acompañado del entonces concejal por el PDA Carlos Vicente de Roux y el Senador Luis Carlos Avellaneda, también de éste partido político, presentaron ante los medios lo que fue llamado el Informe Final de la Comisión de Seguimiento a la Contratación de Bogotá (2010), en donde se describieron las anomalías graves de la contratación de Bogotá. Este documento marcó la ruptura definitiva de Petro con el PDA ${ }^{12}$, pues presentó su renuncia a dicho partido. El mismo documento sirvió de inicio para una serie de investigaciones de carácter penal y disciplinario (por parte de las autoridades competentes) contra varios miembros, tanto de este partido como funcionarios del Distrito, lo que afectó de manera grave la difícil situación que la Alcaldía de Samuel Moreno tenía para entonces.

Es así que al comenzar el 2011, después de la presentación del mencionado informe, se iniciaron medidas que incluyeron la destitución de Moreno Rojas por parte de la Procuraduría General de la Nación y la suspensión para la participación en política que impidió que él pudiera volver al Palacio de Liévano (sede de la Alcaldía Mayor de Bogotá) durante el resto del año de su mandato que terminaría el 31 de diciembre de 2011. El destituido alcalde fue reemplazado en primer lugar por la Ministra de Educación del gobierno de Juan Manuel Santos, María Fernanda Campo y luego por la antigua Secretaria de Gobierno y formula vicepresidencial de Gustavo Petro para las elecciones presidenciales de 2010, Clara López Obregón, quien quedó a cargo de los destinos políticos y administrativos de la Capital durante el último semestre de 2011 (Revista Semana, 8 de junio, 2011).

Las razones que se han ofrecido en relación al tema de la corrupción permiten establecer que, en la coyuntura histórica electoral analizada y siguiendo al catedrático de la Universidad

12. El retiro de Petro del PDA comenzó desde el momento en el cual él propuso la posibilidad de sumarse al "Gobierno de Unidad Nacional" luego de la victoria en primera vuelta de Juan Manuel Santos en las elecciones presidenciales de 2010, lo que generó roces con "Personajes importantes del partido como el senador Jorge Robledo e incluso la ex candidata vicepresidencial de Petro, Clara López, criticaron esa postura y se enfrentaron al ex candidato presidencial", (Batlle, 2011, p.86). 
Complutense, Javier Roiz, la generación de problemas estratégicos de carácter estructural, escaparon de la órbita de acción de las autoridades, lo que desactivó la recepción que tenía la ciudadanía sobre el ejercicio de gobierno distrital, mediante ejercicios permanentes de desmoralización e incapacidad (Roiz, 2003).

De esta manera, se construyó una fuerza en la decisión para las masas electorales de corto plazo que se enfocó en acontecimientos como "cuestiones y evaluaciones comparativas de la actuación pasada de los partidos [en este caso de los candidatos] respecto a asuntos internos" (González y Daría, 1998, p.50) y que fueron aprovechadas por parte de los actores protagonistas del proceso histórico en el sentido de querer buscar un momento simbólico privilegiado que les diera la oportunidad del reconocimiento ante la masa electoral, al generarse un tema político que marcó varias de las líneas de acción de las campañas y en especial para el caso de Petro, por haber sido él quien se encargó de destapar el carrusel, permitió en gran medida que se haya logrado una autonomización de los fines para dicha campaña política.

\section{PROCESO ELECTORAL DE GUSTAVO PETRO URREGO (2011)}

Toda la serie de acontecimientos que se fueron construyendo en torno a al escándalo de la contratación en Bogotá y que incluyó la suspensión de Moreno por parte de la Procuraduría General de la Nación el 3 de mayo de 2011 (El Espectador, 3 de mayo, 2011), fueron aprovechados por los candidatos a la alcaldía en ese momento, debido a la necesidad de enfocar el rol que debían asumir las conductas dentro de la masa electoral. Esto permite demostrar cómo aumentó la intención de voto hacia Petro pues la siguiente encuesta desarrollada por parte del Centro Nacional de Consultoría el 6 de julio le otorgó un 17\% de intención de voto, frente a un 20\% para Peñalosa y un 13\% para Gina Parody.

Petro lograba demostrar que sus denuncias contra la gestión del PDA eran consistentes, pues se respaldaban por una decisión de carácter disciplinario, de esta manera era posible construir la alternativa que permitiera salir de la desesperanza a los electores capitalinos.

Lo anterior también sirvió para que se iniciara la organización de su propio movimiento político denominado Movimiento Progresista (MP), que fue posterior incluso a las intenciones que se venían planteando sobre su participación como candidato a la Alcaldía de Bogotá. Este movimiento político tuvo su lanzamiento el 23 de marzo de 2011 y su primera actividad consistió en la recolección de firmas para el lanzamiento de la candidatura a la Alcaldía del ex concejal de Bogotá Carlos Vicente de Roux ${ }^{13}$, quien acompañó la investigación y la presentación ante los medios del Carrusel de la Contratación.

13. Previo a este lanzamiento, el Concejal De Roux inició una serie de reuniones de trabajo para iniciar su pre candidatura dentro del PDA. 
En ese momento, Petro no planeaba aspirar formalmente como candidato, pues tenía como plan inicial la consolidación del movimiento político (El Espectador, 23 de marzo, 2011) ${ }^{14}$, pero se seguía manteniendo como el referente de las denuncias que se presentaron en Bogotá en torno a la corrupción. Algo que supo aprovechar su campaña al momento de construir los espacios de comunicación que permitieron fortalecer la identidad entre Petro y un nuevo espacio de esperanza para la capital.

Sin embargo, es preciso tener en cuenta una de las fallas generales al momento de construir esta clase de movimientos políticos, pues se considera que "en la mayoría de los casos no son más que candidaturas personales inscriptas mediante firmas por fuera de los partidos" (Basset, 2008, p.8).

Mientras tanto, durante toda la discusión que generó el apoyo que recibía Peñalosa por parte tanto de Uribe Vélez como del PCC y la U, se seguía ejecutando el fortalecimiento de la imagen de anticorrupción que se forjó en torno a Petro cuando, el 18 de agosto de 2011, recibió de parte del Congreso de la República la medalla Luis Carlos Galán Sarmiento (El Tiempo, 20 de agosto, 2011). Situación que se presentó una semana después del cierre de inscripciones a las elecciones y ya en lo que generalmente se denomina campaña electoral. Para ese momento, Petro ya contaba con un $19 \%$ de apoyo en la intención de voto y mantuvo esta tendencia hasta finalizar septiembre.

Dentro del grupo de acontecimientos de la coyuntura electoral se recurrió en apelar a emociones negativas contra Petro por su vinculación con el movimiento guerrillero M-19, situación que desde mucho tiempo atrás había sido utilizada para atacar a cualquier opositor del gobierno nacional, mediante la repetición constante de los vínculos entre los grupos subversivos y las acciones consideradas como terroristas, así como la financiación por medio del narcotráfico de la toma del Palacio de Justicia en 1985, circunstancia que en muchos casos se descontextualiza con el fin de enfocarse únicamente en aquellos acontecimientos que de manera más fuerte pueden modificar la percepción sentimental que se tenga sobre determinado actor político.

Al encontrarse Petro en una posición de mayor comodidad durante las últimas semanas de la campaña política, sobre él comenzaron a desarrollarse dichas acciones. Además de la mencionada por su vinculación con el extinto M-19, se comenzó a criticar varias de sus acciones y de sus miembros en su equipo de trabajo. Se iniciaron críticas de parte de algunos sectores de la Izquierda por el hecho de haber recibido financiamiento para su campaña por

14. Finalmente, se permitió que De Roux fuera el cabeza de lista del Movimiento Progresista al Concejo de Bogotá. Él, inclusive se aventuró a postular su nombre como precandidato dentro del PDA, antes de renunciar para poder conformar el MP. 
parte de algunos de los grupos económicos más importantes del país, como el caso del Grupo Bolívar (que maneja, entre otros al Banco Davivienda y la Compañía de Seguros Bolívar), el Grupo Colpatria; así como la multinacional francesa Casino por intermedio la compañía de Almacenes Éxito (La Silla Vacía, 28 de octubre, 2011). Situación que para algunos se volvió en una paradoja pues estaría en contra de algunos de sus proyectos políticos y sus ideas en temas económicos al recibir el aporte de estos grupos, esencialmente en aspectos como el impulso que debía tener la capital en vísperas de la aprobación del Acuerdo de Promoción Comercial con EEUU.

Este último punto fue utilizado como parte de una estrategia desde las bases del PDA quienes trataron de relacionar a Petro con alguien vinculado fuertemente a raíces de corte neoliberal, como el plan que propuso de efectuar el cobro de peajes dentro de la ciudad en zonas de alta congestión, situación que llevó a que el Senador Jorge Enrique Robledo calificará así: "Los peajes urbanos son neoliberalismo, FMl y consenso de Washington. Esos son sus orígenes, en nada afectos a las ideas democráticas" (El Espectador, 2 de diciembre, 2011, párr. 6).

Continuando con el tema de investigación, es preciso mencionar que durante la última semana antes de las elecciones ya era evidente que determinadas masas electorales tenían una tendencia clara frente a un determinado candidato y la tendencia era poco probable que pudiera revertirse. La última encuesta del Centro Nacional de Consultoría fue el 28 de octubre y en ella se observó un porcentaje de 30\% para Petro, 21\% a Peñalosa y 15\% para Parody. Las acciones que se desarrollaron en esos últimos días simplemente modificaron un poco la intención de voto, más sin embargo la tendencia quedó establecida de esa manera.

El 30 de octubre, día de las elecciones, los resultados reflejaron, como sucede en pocas ocasiones, concordancia con lo establecido en dicha encuesta. Petro fue el ganador al obtener un total de 723.157 votos, un porcentaje del $32.22 \%$. El segundo lugar correspondió a Peñalosa con 560.590 (24.98\%) y Parody logró el tercer lugar con 376.154 votos, que representan un 16.76\% (Registraduría Nacional del Estado Civil, 5 de diciembre, 2011).

Estos resultados también muestran el quiebre de una tendencia en relación a la participación en la jornada electoral por parte del electorado capitalino, pues es la primera vez que es elegido un Alcalde con una votación menor a la de las elecciones inmediatamente anteriores: en las elecciones de 2003, Garzón obtuvo 797.466 (Registraduría Nacional del Estado Civil, 30 de marzo, 2004) votos y para el año 2007, Moreno Rojas alcanzó la cifra de 920.013 votos. Esto se vio reflejado en parte porque también, a diferencia de otras contiendas en donde se mantenía la competencia entre dos candidatos y una opción de tercería, en esta ocasión otros candidatos también lograron alcanzar niveles de votación considerables, como fue el cuarto lugar que obtuvo Carlos Fernando Galán con una votación de 285.263 votos, para un total del $12.71 \%$. 
En general, los niveles de abstención se mantuvieron estables ${ }^{15}$, lo que permite establecer que en términos de conducta del voto existe un bloque de abstencionistas duros que no buscan el interés de participar en ningún tipo de proceso electoral, siendo significativamente menor aquellos que se pueden considerar abstencionistas intermitentes, para los cuales, como lo menciona Marco Fournier (2002), la definición de su voto se adecua más a condiciones de carácter coyuntural que les impulsa a movilizarse políticamente a favor o en contra de determinado candidato.

\section{CONCLUSIÓN}

Como ha sucedido en la historia política reciente de Colombia, los procesos políticos se han construido mediante acontecimientos coyunturales que permiten que las masas electorales comiencen a configurar el diseño de una compatibilidad política que se base en la satisfacción de sus necesidades y la recuperación en los escenarios de crisis, mediante la articulación con diversos acontecimientos que permitieron impulsar su intención de voto hacia determinada opción, situación que reafirma lo establecido por Francisco Gutiérrez en relación con "el aumento de la volatilidad electoral y de la franja de electores que no se guían por un membrete partidista para votar" (2006, p.67).

Durante los últimos años, el triunfo de la Izquierda en la capital ha generado espacios de comparación con acontecimientos similares en otros países de América Latina, más sin embargo no se ha logrado configurar las razones concretas de la ruptura y la crisis en la que se ha visto asumida.

En el caso de las últimas elecciones, efectuadas en el año 2011, de acuerdo con lo expresado por diversos medios, la estrategia anticorrupción de Petro fue el punto de anclaje con muchos de sus electores, así como el apoyo que muchos sectores políticos de la Izquierda política, como ciertos grupos políticos dentro del PDA, lograron impulsar su candidatura en sectores populares (La Silla Vacía, 30 de octubre, 2011), a diferencia de la anhelada unidad que consideraban en su primer momento, cuando fue posible el triunfo de Garzón, concretándose ese ideal durante el periodo de Moreno.

Sin embargo, paradójicamente, en ese mismo momento se comenzó a observar la crisis que vivía la Izquierda nacional, pues no se logró mantener dentro del partido PDA a Garzón luego de terminar su periodo y, posteriormente, comenzó el proceso de salida de Petro y la posterior suspensión sobre Moreno.

15. Para el año de 2003, la abstención fue de un 56,49\%; en el año 2007 se obtuvo una tasa de abstención de 52.17\% y en las pasadas elecciones la abstención llegó al 52.59\%. No debe dejarse a un lado que también ha aumentado en ese periodo de tiempo el censo electoral. 
En este momento en particular, vinculado a las diversas críticas que ha sufrido Petro como Alcalde Mayor y el proceso de destitución que inició la Procuraduría General de la Nación en su contra, no se puede observar una condición optimista para la Izquierda.

Además, en las elecciones de autoridades nacionales del año 2014, volvieron al ruedo político diversos movimientos de afiliación de Izquierda, con una gran variedad de matices, como es el caso de la vuelta al ruedo electoral de la UP, la alianza entre el MP y el PV con el fin de construir el partido Alianza Verde (AV), y el retiro de miembros del PC del PDA como consecuencia de la creación del movimiento político Marcha Patriótica.

Lo anterior también debe observarse en relación con los cambios electorales para las próximas elecciones de autoridades nacionales (Senado y Cámara de Representantes), pues se ha establecido un nuevo umbral electoral del $3 \%$ de los votos válidos para poder mantener la personería jurídica, situación que afecta la nueva diversidad y disgregación de la izquierda nacional, siendo posible la perdida de reconocimiento legal para estos movimientos y, por ende, afectando su configuración con miras a procesos electorales posteriores.

\section{REFERENCIAS}

Alcaldía Mayor de Bogotá (2001). Comportamiento electoral en Bogotá 1982 - 1997, Bogotá, Instituto para el desarrollo de la democracia Luis Carlos Galán, II.

Alted, A. y Sánchez, J. (2006). Métodos y Técnicas de Investigación en Historia Moderna e Historia Contemporánea, Madrid: Editorial Centro de Estudios Ramón Areces.

Ayala, C. (1995). Nacionalismo y Populismo. ANAPO y el Discurso Político de la Oposición en Colombia: 1960 - 1966, Bogotá, Universidad Nacional de Colombia.

Batlle, M. (2011). "La difícil tarea de seleccionar un candidato: cambios y continuidades en las estrategias de selección en los partidos colombianos (1990-2010)", en, Basset, Yann et al, Elección 2010: Partidos, Consultas y Democracia Interna, Bogotá, Universidad Externado de Colombia, pp.51-105.

Bernal, C. (2006). Metodología de la Investigación. Para Administración, Economía, Humanidades y Ciencias Sociales, México, Pearson Educación de México.

Caracol Radio (5 de marzo, 2007). El Polo Democrático y la consulta sobre candidato a la Alcaldía de Bogotá, Recuperado de http://www.caracol.com.co/audio_programas/archivo_de_ audio/el-polo-democratico-y-la-consulta-sobre-candidato-a-la-alcaldia-de-bogotahab̆lanjorge-robledo-y-maria-ema-mejia/20070305/oir/398324.aspx consultado el 20-06-2014. 
(1 ${ }^{\circ}$ de febrero, 2011). Esta es la Historia del "Carrusel de la Contratación", Recuperado de http://www.caracol.com.co/noticias/bogota/esta-es-la-historia-del-carruselde-contratacion/ 20110201/nota/1419470.aspx consultado el 21-06-2014.

Cepeda, F. (s.f.). La Reforma Política en Colombia, Recuperado de www.icpcolombia.org/ archivos/documentos/reformapoliticall.doc Consultado el 18-06-2014.

Dalsgaard, A. (2009). Bogota change, Recuperado de http://vimeo.com/25521307 consultado el 17-06-2014.

Dávila, A. (1992). “¿Del bipartidismo a un Nuevo Sistema de Partidos? Crisis, Constituyente y Reconstrucción de un Orden Político Democrático en Colombia", en, América Latina hoy, 3, pp, 35-42.

De Gyves, F. (s.f.). Web Mining: Fundamentos Básicos. Doctorado en Informática y Automática, Salamanca, Universidad de Salamanca, Recuperado de http://zarza.usal.es/ fgarcia/ doctorado/iweb/05-07/Trabajos/WMINING.pdf consultado el 18-06-2014.

De Roux, C. y Avellaneda, L. (2010). Informe final de la comisión de seguimiento a la contratación de Bogotá, Bogotá, Recuperado de http://www.carlosvicentederoux.org/apcaa-files/b3103a6119a79c0bec1c7e4eb88dc340/Informe\%20Comisi\%F3n\%20de\%20 Seguimiento\%20a\%20la\%20Contrataci\%F3n\%20de\%20Bogot\%E1.pdf Consultado el 2106-2014.

El Espectador (23 de marzo, 2011). Petro presentó a Carlos Vicente de Roux como candidato a la Alcaldía, Recuperado de http://www.elespectador.com/noticias/politica/petro-presentocarlos-vicente-de-roux-candidato-alcaldi-articulo-258686 Consultado el 22-06-2014.

(3 de mayo, 2011). Samuel Moreno, Suspendido por Tres Meses del Cargo, Recuperado de http://www.elespectador.com/noticias/bogota/samuel-morenosuspendido-tres-meses-del-cargo-articulo-266979 Consultado el 22-06-2014.

(2 de diciembre, 2011). Jorge Robledo arremetió contra 'peajes' de Gustavo Petro, Recuperado de http://www.elespectador.com/noticias/politica/jorge-robledoarremetio-contra-peajes-de-gustavo-petro-articulo-314652 Consultado el 21-06-2014.

El Tiempo (7 de agosto, 1997). La alcaldía, con 16 pretendientes, Recuperado de http://www. eltiempo.com/archivo/documento/MAM-648098 Consultado el 14-06-2014.

(13 de noviembre, 2004). "El 28, el candidato del PDI", Recuperado de http://www. eltiempo.com/archivo/documento/MAM-1587602 Consultado el 21-06-2014 
(26 de octubre, 2007). Presidente Uribe Insistió Hoy en sus Alusiones contra Samuel Moreno, Candidato a la Alcaldía de Bogotá, Recuperado de http://www.eltiempo.com/ archivo/documento/CMS-3785820 Consultado el 19-06-2014

(20 de agosto, 2011). Petro, condecorado por lucha contra la corrupción, Recuperado de http://www.eltiempo.com/archivo/documento/MAM-4765709 Consultado el 22-062014

Estrada, J. (2008). "Proyectos de izquierda y gobiernos alternativos: un análisis de la experiencia colombiana reciente", en, Stolowicz, B, (Coord.), Cobiernos de izquierda en América Latina: un balance político, Bogotá, Ediciones Aurora, pp.285-321.

Fazio, H. (1998). "La historia del tiempo presente: Una historia en construcción", en, Historia crítica, 17, julio-diciembre, pp.47-57.

(2007). "La historia del tiempo presente y la modernidad mundo", en, Historia crítica, 34, julio - diciembre, pp.184-207.

Flórez, J. (2011). "Constitución Política de 1991: descentralizar para gobernar", en, en, Historia institucional de la Alcaldía Mayor de Bogotá, Bogotá, Alcaldía Mayor de Bogotá, Universidad del Rosario, T. II, pp.155-202.

Fournier, M. (2002). "Una tipología de los electores", en, Revista de ciencias sociales, IV(IV), pp.9-18.

González, M. y Daría, M. (1998). "Predicción de la conducta del voto: personalidad y factores psicopolíticos", en, Psicología política, 17, pp, 45-78.

Gutiérrez, F. (1995). "Tendencias de cambio en el sistema de partidos: el caso de Bogotá", en, Análisis Político, 24, Enero - abril, pp, 73-83.

(2006). ¿Lo que el viento se llevó?: Los partidos políticos y la democracia en Colombia (1958-2002), Bogotá, Grupo Editorial Norma.

Hamburger, Á. (2014). “El socialismo del siglo XXI en América Latina: Características, desarrollos y desafíos", en, Revista de relaciones internacionales, estrategia y seguridad, 9(1), pp, 131154.

La Silla Vacía (23 de noviembre, 2010). Luis Eduardo Garzón. Presidente del Partido Verde, Recuperado de http://www.lasillavacia.com/perfilquien/20070/luis-eduardo- Consultado el 15-06-2014. 
(28 de octubre, 2011). ¿A quién financian los cacaos?, Recuperado de http:// lasillavacia.com/historia/quien-financian-los-cacaos-29137 Consultado el 22-062014.

(30 de octubre, 2011). Bogotanos premian la lucha anticorrupción de Petro, Recuperado de http://lasillavacia.com/historia/bogotanos-premian-lucha-anticorrupcionde-petro-29204. Consultado el 22-06-2014.

Landi, Ó. (1995). Óscar Landi, "Outsiders, Nuevos Caudillos y Media Política", en, Grompone, R, (ed.), Instituciones Políticas y Sociedad, Lima, Instituto de Estudios Peruanos, pp.204-217.

Lozano, M. (2008). "Los procesos de subjetividad y participación política de estudiantes de psicología de Bogotá", en, Diversitas. Perspectivas en psicología, 4(2), julio-diciembre, pp.345-357.

Mendoza, A. (2006). "Colombia: Estado regional unitario", en, Revista de relaciones internacionales, estrategia y seguridad, 1(1), pp.55-67.

Ministerio del Interior, (1999). Informe Consultoría Internacional Sobre la Reforma Política. Reforma Política. Un Propósito de Nación, 17, Bogotá.

Otálora, C. (s.f.) Elecciones y prácticas políticas en Bogotá. Un Objeto de Estudio en Construcción, Bogotá, Universidad Nacional de Colombia.

Radnitzky, G. "Hacia una Teoría de la Investigación que no es ni Reconstrucción Lógica ni Psicología o Sociología de la Ciencia", en, Teorema. Revista Internacional de Filosofía, 3(23), pp.197-264.

Ramalho, A. (2007). "Evolução do sistema internacional contemporão: implicações para a inserção internacional do Brasil", en, Alvear, L, y Octavio, A, (coord.), Sistema político brasileiro: uma introdução, Rio de Janeiro, Konrad Adenauer Stiftung y Editora UNESP, pp.435-449.

Registraduría Distrital (1988). Estadísticas distritales, Bogotá, Imprenta Nacional.

(1990). Estadísticas distritales, Bogotá, Imprenta Nacional.

Registraduría Nacional del Estado Civil (6 de julio, 2002). Informe de votación, Recuperado de http://web.registraduria.gov.co/2002PRP1/e/dpresidente01.htm Consultado el 15-062014. 
(30 de marzo, 2004). Informe de votación. Votación para el municipio de Bogotá, D.C., Recuperado de http://web.registraduria.gov.co/2003CC/e/val16001.htm?1 Consultado el 21-06-2014.

(5 de diciembre, 2011). Resultados finales de alcaldía. Elecciones 30 de octubre de 2011, Recuperado de http://w3.registraduria.gov.co/escrutinio/resultados Consultado el 19-06-2014.

(s.f.), Boletín informativo: Escrutinios alcalde. Elecciones: octubre 28 de 2007, Recuperado de http://web.registraduria.gov.co/reselec2007_esc/1028/x.html Consultado el 19-06-2014.

Revista Semana (23 de diciembre, 2002). Los dinosaurios inextinguibles, Recuperado de http:// www.semana.com/nacion/articulo/los-dinosaurios-inextinguibles/55692-3 Consultado el 23-06-2014

(8 de junio, 2011). Clara López, nueva alcaldesa (e) de Bogotá, Recuperado de http://www.semana.com/nacion/articulo/clara-lopez-nueva-alcaldesa-e-bogota/241060-3 Consultado el 23-06-2014.

Rodríguez, C. (2008). "Colombia. La nueva izquierda: orígenes, características y perspectivas", en, Chávez, D, et al, La nueva izquierda en América Latina, Madrid, Los libros de la catarata, pp, 189-220.

Roiz, J. (2003). La recuperación del buen juicio. Teoría política en el siglo XX, Madrid, Editorial foro interno.

YouTube (2007). SamuEl (sic) el compra votos, Recuperado de https://www.youtube.com/ watch?gl=ES\&hl=es\&v=85wK5fOw9Bo consultado el 20-06-2014. 